ACCEPTED MANUSCRIPT

\title{
T1 and extracellular volume fraction mapping in cardiac magnetic resonance: estimation of accuracy and precision of a novel algorithm
}

To cite this article before publication: Cristian Borrazzo et al 2019 Phys. Med. Biol. in press https://doi.org/10.1088/1361-6560/aafcca

\section{Manuscript version: Accepted Manuscript}

Accepted Manuscript is "the version of the article accepted for publication including all changes made as a result of the peer review process, and which may also include the addition to the article by IOP Publishing of a header, an article ID, a cover sheet and/or an 'Accepted

Manuscript' watermark, but excluding any other editing, typesetting or other changes made by IOP Publishing and/or its licensors"

This Accepted Manuscript is @ 2018 Institute of Physics and Engineering in Medicine.

During the embargo period (the 12 month period from the publication of the Version of Record of this article), the Accepted Manuscript is fully protected by copyright and cannot be reused or reposted elsewhere.

As the Version of Record of this article is going to be / has been published on a subscription basis, this Accepted Manuscript is available for reuse under a CC BY-NC-ND 3.0 licence after the 12 month embargo period.

After the embargo period, everyone is permitted to use copy and redistribute this article for non-commercial purposes only, provided that they adhere to all the terms of the licence https://creativecommons.org/licences/by-nc-nd/3.0

Although reasonable endeavours have been taken to obtain all necessary permissions from third parties to include their copyrighted content within this article, their full citation and copyright line may not be present in this Accepted Manuscript version. Before using any content from this article, please refer to the Version of Record on IOPscience once published for full citation and copyright details, as permissions will likely be required. All third party content is fully copyright protected, unless specifically stated otherwise in the figure caption in the Version of Record.

View the article online for updates and enhancements. 


\section{T1 and extracellular volume fraction mapping in cardiac magnetic resonance: estimation of accuracy and precision of a} novel algorithm

Cristian Borrazzo ${ }^{\mathrm{a}, \mathrm{b}^{*}}$, Massimiliano Pacilio ${ }^{\mathrm{b}}$, Nicola Galea $^{\mathrm{c}}$, Enrico Preziosi ${ }^{\mathrm{d}}$, Marco Carni ${ }^{\mathrm{b}}$, Marco Francone $^{\mathrm{c}}$, Carlo Catalano ${ }^{c}$, Iacopo Carbone ${ }^{c}$

a Department of Public Health and Infectious Disease, "Sapienza” University of Rome, Viale del Policlinico 155, 00161 Rome, Italy

${ }^{\mathrm{b}}$ Department of Medical Physics, Azienda Ospedaliera Universitaria Policlinico Umberto I, Viale del Policlinico 155, 00161 Rome, Italy

cDepartment of Radiological, Oncological and Anatomo-Pathological Sciences, "Sapienza" University of Rome, Viale del Policlinico 155, 00161 Rome, Italy

${ }^{\mathrm{d}}$ Department of Physics and NAST Center, "Tor Vergata" University of Rome, Via della Ricerca Scientifica 1, Rome, 00179 Italy.

*Corresponding author (cristian.borrazzo@uniroma1.it) 


\section{ABSTRACT}

Magnetic resonance imaging is effective for non-invasive detection of myocardial diseases by Extracellular Volume Fraction (ECV) estimation. A new methodology for T1 and ECV mapping is tested in this work, comparing results with other well-consolidated methods. The associated level of uncertainty for data was also estimated, to assess the reliability of the technique. A phantom with known T1 values was used for reference, and 60 subjects (40 controls, 20 diseased patients) were examined, using the Modified Look-Locker Inversion-Recovery (MOLLI) method. Obtained T1 data were studied in terms of accuracy (difference with reference T1), paired comparisons with other methods, and Gamma-tool analysis with tolerances criteria of $4.21 \mathrm{~mm}$ for distance-to-agreement, and between 2-5\% for T1 difference. Accuracy and precision of the $\mathrm{T} 1$ mapping was analysed by phantom measurements, and the uncertainty of the ECV was estimated by T1 error propagation. Differences (in paired comparisons) of $\mathrm{T} 1$ datasets were not significant neither for statistical tests, nor for Bland-Altman analysis. T1 accuracy was between $-12 \%$ and $-1 \%$ across methods, slightly better for the tested method (mean accuracy in the T1 range of interest better than $2 \%$ ). The Gamma analysis confirm slightly better results for the tested method than other methodologies. The new method improves the computational efficiency by a factor of 25 (at least), revealing to be also more suitable for Big Data-related applications. 


\section{Introduction}

Late Gadolinium Enhanced (LGE) Cardiac Magnetic Resonance (CMR) is a diagnostic reference for focal myocardial fibrosis, whose importance grew further after the development and optimisation of the InversionRecovery (IR) sequence [1-3]. T1 mapping can also detect diffuse myocardial disease [4-6], but the T1 of the normal myocardium cannot be measured directly in diseased patients. Data for various tissues were reported recently in literature [7], in most cases interpolating signal data by the Levenberg Marquardt (LM) álgorithms [8]. However, the Extracellular Volume Fraction (ECV, defined as the fraction of myocardial tissue not occupied by cells) has proven to be a more accurate and reproducible biomarker for myocardial disease [9, 10], and can be determined calculating the myocardium/blood partition coefficient by mapping $\mathrm{T} 1$ before and after the Gadolinium administration. The uncertainty and accuracy of T1 values affects the ECV estimation. The level of precision (i.e., space and time reproducibility) and accuracy of T1 measurement, are generally influenced by many components, such as technical factors (e.g. sequence-related, partial volume effects), physiological factors (e.g., breath-holds, cardiac motion, flowing blood, and haematocrit), procedural errors (e.g., sequence-related parameters, repeatability of the scanner acquisition, and data fitting), and assumptions related to the contrast-agent [11]. A new, time-sparing, procedure to obtain the ECV map was recently described [12], in which a Nonlinear Least Squares (NLS) problem is solved to fit a five-parameter model to a dataset. In this method, the computational complexity of the problem was reduced to a search over one dimension. The algorithm concurrently restored polarity if only signal magnitudes are available. In the present work, the reliability of this method is further explored, investigating precision (space and time reproducibility) and accuracy of $\mathrm{T} 1$, calculating the propagated error for ECV and comparing the error range with the interpatient variability. Firstly, the results obtained with the new methodology are compared with those from wellconsolidated methods (previously published [13], and suggested by the scanner manufacturer [14]). Secondly, the error contributions to $\mathrm{T} 1$ values due to the fitting procedure and measurement repeatability are estimated (with phantom scans). Finally, the ECV values are calculated for a large cohort of patients (40 patients with known or suspected heart disorder and 20 healthy controls), comparing the error range with the inter-patient variability. 


\section{Materials and Methods}

\section{Numerical and experimental phantom}

Eight flasks ( $3 \mathrm{~cm}$ of diameter and $4 \mathrm{~cm}$ high), containing $2 \%$ of agarose gel (Sigma-Aldrich $\odot$ ) and $0.5 \%$ of $\mathrm{NaCl}$ (to mimic the tissue electrical conductivity), were prepared varying the concentration of $\mathrm{CuSO}_{4}$ (SigmaAldrich () between $0.25 \mathrm{mM}$ and $2 \mathrm{mM}$, obtaining T2 around $50 \mathrm{~ms}$ (i.e. the normal myocardium value) and T1 values in the range $250-1000 \mathrm{~ms}$. A ninth flask was a vial filled with $0.5 \%$ agarose gel and $0.025 \mathrm{mM}$ of $\mathrm{CuSO}_{4}$, to mimic blood $\mathrm{T} 1$ and $\mathrm{T} 2$ (about $1500 \mathrm{~ms}$ and $200 \mathrm{~ms}$, respectively) [15]. The flásks were tied among them, and imaged with a 1.5 T MR tomograph (Magnetom Avanto, Siemens Healthcare, at Erlangen Germany). The reference T1 values of the flasks were measured with an IR (gold-standard) sequence using 25 inversion times (TIs) in the range 100-2500 ms and interpolating signal data with the LM algorithm, (also, T2 values were measured with a standard spin-echo pulse sequence using 10 echo times, but data are not shown). The T1 for each test object was determined from the T1 map as/mean value in a ROI, covering about $80 \%$ of the cross-sectional area of the selected flask. A numerical phantom was generated with MATLAB (R2009b, version 7.9.0.529), oversampling the acquisition matrix (from a pixel size of $1.41 \times 1.41 \mathrm{~mm}^{2}$ to $0.71 \times 0.71$ $\mathrm{mm}^{2}$ ) and assigning to each flask the corresponding reference T1 value (Figure 1). 
The phantom was scanned also with MOLLI (Modified Look-Locker Inversion-recovery) sequences for cardiac imaging, provided by the scanner manufacturer, comparing so measured $\mathrm{T} 1$ values with the reference values. A pre-contrast sequence scheme of 5(3)3 (i.e., five images are acquired starting from the first TI, and after a three-cardiac cycle pause, three additional images are acquired starting from the sixth TI), or 4(1)3(1)2 for the post-contrast sequence, was chosen to decrease the scanning time with comparable accuracy and precision [14]. Other sequence parameters were: matrix size $=218 \times 256$, voxel size $=1.41 \times 1.41 \times 8 \mathrm{~mm}^{3}$, $\mathrm{TR} / \mathrm{TE}=2.8 / 1.12 \mathrm{~ms}$, and Flip Angle=35 . TI values pre-defined by the scanner manufacturer were used [14]. Image analysis and data interpolation for the MOLLI sequences are reported in the following.

\section{Image analysis and fitting procedures for MOLLI sequences}

Firstly, median filtering and thresholding with a variable cut-off were used to remove salt-and-pepper and random noise of the images. Data interpolations were performed by the Fast Nonlinear Least Squares (FNLS) algorithm implemented in Altabella et al. [12], obtaining pixel-wise mapping of $\mathrm{T} 1$. The following fitting model (including also $\mathrm{T} 1 *$ correction) was used:

$$
\begin{aligned}
& S(i, j, T I)=A(i, j)+B(i, j) \cdot e^{-\frac{T I}{T 1^{*}(i, j)}} \\
& T 1(i, j)=T 1^{*}(i, j) \cdot\left(\frac{B(i, j)}{A(i, j)}-1\right),
\end{aligned}
$$

where A represent the scaling factor for signal intensity, B reflect the quality of the inversion, and $\mathrm{T} 1 *$ is the apparent T1.The aim is to find the best fitting T1(i, $j)$ in the least squares sense (i.e. in terms of sum of squares) [12]. Equation 1 was solved using a reduced dimension approach, separating the unknown variables in $\mathrm{S}(\mathrm{i}, \mathrm{j}, \mathrm{TI})$, as also proposed previously by Barral et al. [16]. Using this approach, $\mathrm{T} 1 \mathrm{can}$ be found through one-dimensional search, without need of initial parameter estimation as a global search in the selected range of values is performed [12]. The results of FNLS were compared with those obtained from the method of Messroghli et al. [13] and from the scanner manufacturer [14]. Both of them use the LM algorithm, with a difference in the stop conditions for the iterations. Hereinafter, the three methods will be referred to as FNLS, MM and MS respectively. 
The accuracy associated to mean T1 measured by MOLLI (with the same ROI used for IR acquisition, see phantom section) is defined as follows:

$$
\mathrm{T} 1 \operatorname{accuracy}(\%)=\frac{\mathrm{T} 1_{\mathrm{MOLLI}}-\mathrm{T} 1_{\mathrm{IR}}}{\mathrm{T} 1_{\mathrm{IR}}} \cdot 100 .
$$

Spatial reproducibility was estimated as the Standard Deviation (SD) in the ROI. Time reproducibility (testretest reproducibility) was assessed repeating the MOLLI acquisition once a day, for 9 days, calculating the associated SD for the mean T1. The T1 uncertainty was estimated assuming that the total uncertainty consists in the composition of two independent random errors normally distributed, so combining the time-dependent and spatial-dependent term into a quadratic sum.

The Gamma-tool analysis, commonly used in radiotherapy to study the pixel-wise agreement between measured and reference spatial distribution of absorbed dose [17, 18], was here implemented (in MATLAB) to perform paired comparisons between reference (IR) and tested (MOLLI) T1 maps. The reference T1 map was obtained by down-sampling the numerical phantom aforementioned to the experimental acquisition matrix (i.e., matrix size $218 \times 256$ and pixel size $1.41 \times 1.41 \mathrm{~mm}^{2}$ ). The tolerance criteria in terms of Distance to Agreement (DTA) and pixel-by-pixel Difference (D) were set $[17,18]$. The DTA tolerance was selected as 3 pixels $(4.23 \mathrm{~mm})$, as the general rule states that the sampling pitch (i.e. the pixel size) should be less than or equal to one third of the DTA tolerance [18]. The D tolerance was varied in the range 2-5\%. Gamma $(\gamma)$ values higher than 1 indicate failure to comply with the tolerances adopted.

\section{Patient population and data acquisitions}

40 patients with known/suspected heart disorder ( 5 dilated cardiomyopathy, 5 pulmonary hypertension, 5 hypertrophic cardiomyopathy, 5 non-compacted cardiomyopathy, 15 myocarditis, and 5 cases with other cardiac conditions) and 20 healthy controls were examined. The local ethics committee approved the study, and all subjects provided written informed consent. Apical, basal and mid-ventricular slice were acquired for each subject, pre- and post-injection (15 min later) of a Gd-BOPTA bolus (gadobenate dimeglumine, 0.1 $\mathrm{mmol} / \mathrm{kg}$ ) [19], using the pre- and post-contrast MOLLI sequences described above ("Numerical and experimental phantom"). Signal images referred to various TI were systematically motion-corrected and coregistrated, using software provided by the manufacturer [20]. 


\section{ECV calculation}

The ECV maps were calculated from the pre- and post-contrast T1 map, applying the following expression:

$$
\operatorname{ECV}(\%)=(1-\mathrm{Hct}) \cdot \frac{\Delta \mathrm{R} 1_{\text {myo }}}{\Delta \mathrm{R} 1_{\text {blood }}} \cdot 100
$$

where $\quad \Delta \mathrm{R} 1_{\text {myo }}=\left(1 / \mathrm{T} 1_{\text {post-myo }}-1 / \mathrm{T} 1_{\text {pre-myo }}\right)$ and $\Delta \mathrm{R} 1_{\text {blood }}=\left(1 / \mathrm{T} 1_{\text {post-blood }}-1 / \mathrm{T} 1_{\text {pre-blood }}\right)$ are the longitudinal relaxation rate (R1) enhancement for myocardium and blood pool, respectively, and Hct/s the patient hematocrit (i.e., the ratio between red blood cell volume and total blood volume). The ECV maps were calculated by the FNLS method (the MM and MS methods do not calculate directly ECV, but just T1 maps), performing also preliminary affine co-registration of pre-contrast and post-contrast T1 map [12]. Patient myocardial ECV was also used to compare several methods. Patient myocardial ECV was obtained as an average in a ROI delineating the myocardium region, drawn on the pre-contrast T1 map excluding pixels with values higher than $1250 \mathrm{~ms}$, and overimposed on the ECV map (see Ref. 16 for a more detailed description).

\section{Statistical analysis}

The statistical tests were performed with MATLAB, using paired comparisons between the different methods (FNLS, MS and MM) by Bland-Altman analysis, Kruskal-Wallis test, and Student's t-test, using Graph Pad Prism software (ver. 6, GraphPad Software, La Jolla California USA). The level of statistical significance chosen was p-value $<0.05$. For each method, patient ECVs were represented in boxplots reporting median, mean, interquartile range of $25-75 \%$, and outliers, whereas the ECV relative error was calculated by propagation of $\mathrm{T} 1$ errors (spatial and time reproducibility) and represented as a histogram.

\section{Results}

\section{Phantom studies}

Differences (in paired comparisons) of mean T1 datasets were not significant neither for Student's t-test, nor for Kruskal-Wallis test (always p>0.20). Analogously, paired comparison for mean T1 with Bland-Altman plots did not evidence trend differences (Figure 2 reports an example of Bland-Altman plots for the 4(1)3(1)2 MOLLI sequence). 

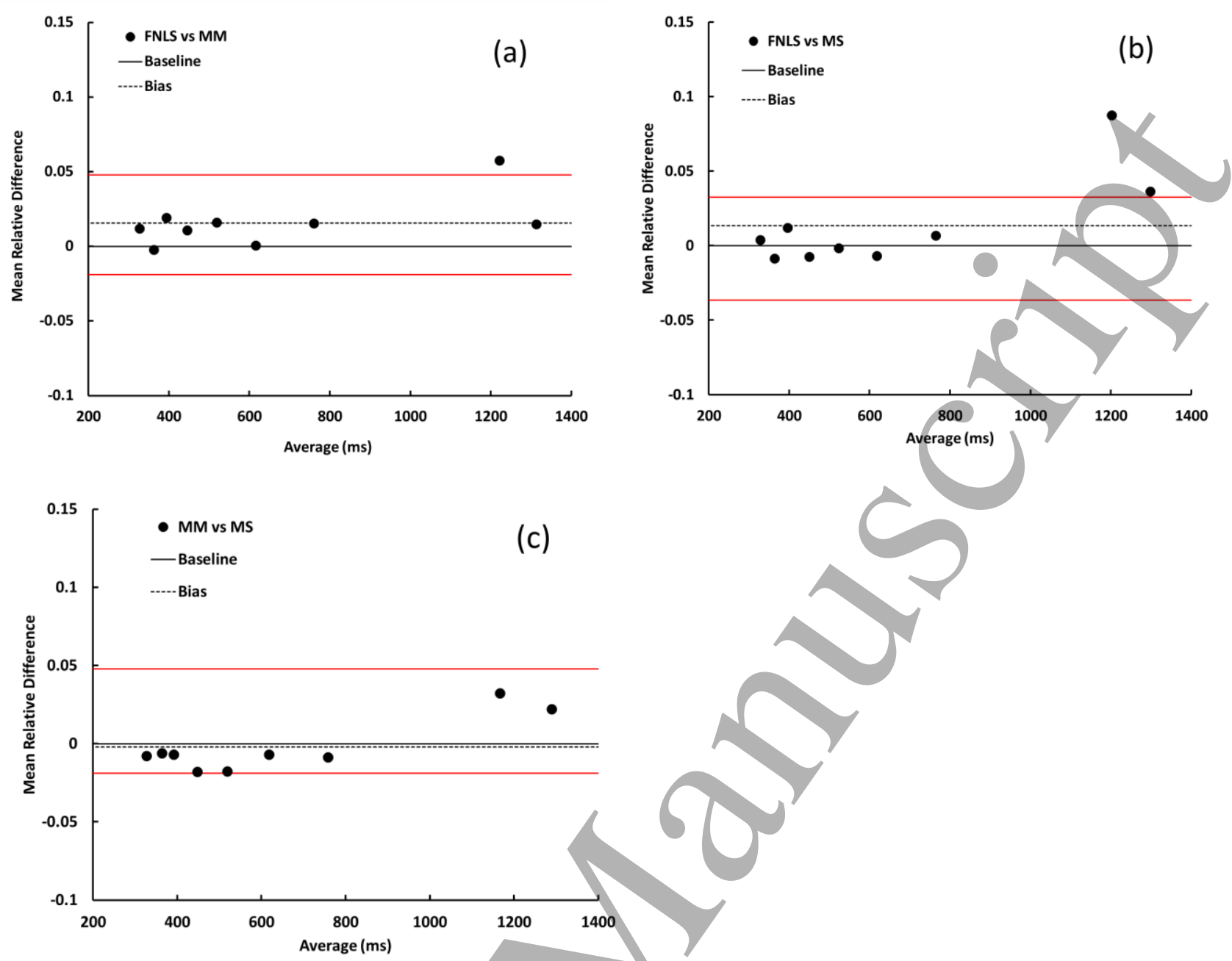

Figure 2. Bland-Altman plots showing paired comparisons for the MOLLI sequence 4(1)3(1): FNLS vs MM (a), FNLS vs MS (b), and MM vs MS (c). The $95 \%$ confidence interval (red lines) and bias (dotted lines) are also displayed.

The T1 accuracy (Equation 4) for each method is reported in Figure 3 for the 4(1)3(1)2 and 5(3)3 MOLLI sequence. The differences were between $-12 \%$ and $-1 \%$ across methods, with average differences for the 4(1)3(1)2 sequence of $-3.2 \%$ for MM, $-1.7 \%$ for FNLS and $-2.9 \%$ for MS, and for the $5(3) 3$ sequence of $2.2 \%$ for MM, $-1.6 \%$ for FNLS and $-2.5 \%$ for MS. The FNLS showed the best computational efficiency, calculating T1 maps in $18 \mathrm{~s}$ with a standard desktop PC, whereas the computational time was $822 \mathrm{~s}$ and $471 \mathrm{~s}$ for the MM and MS method, respectively. 

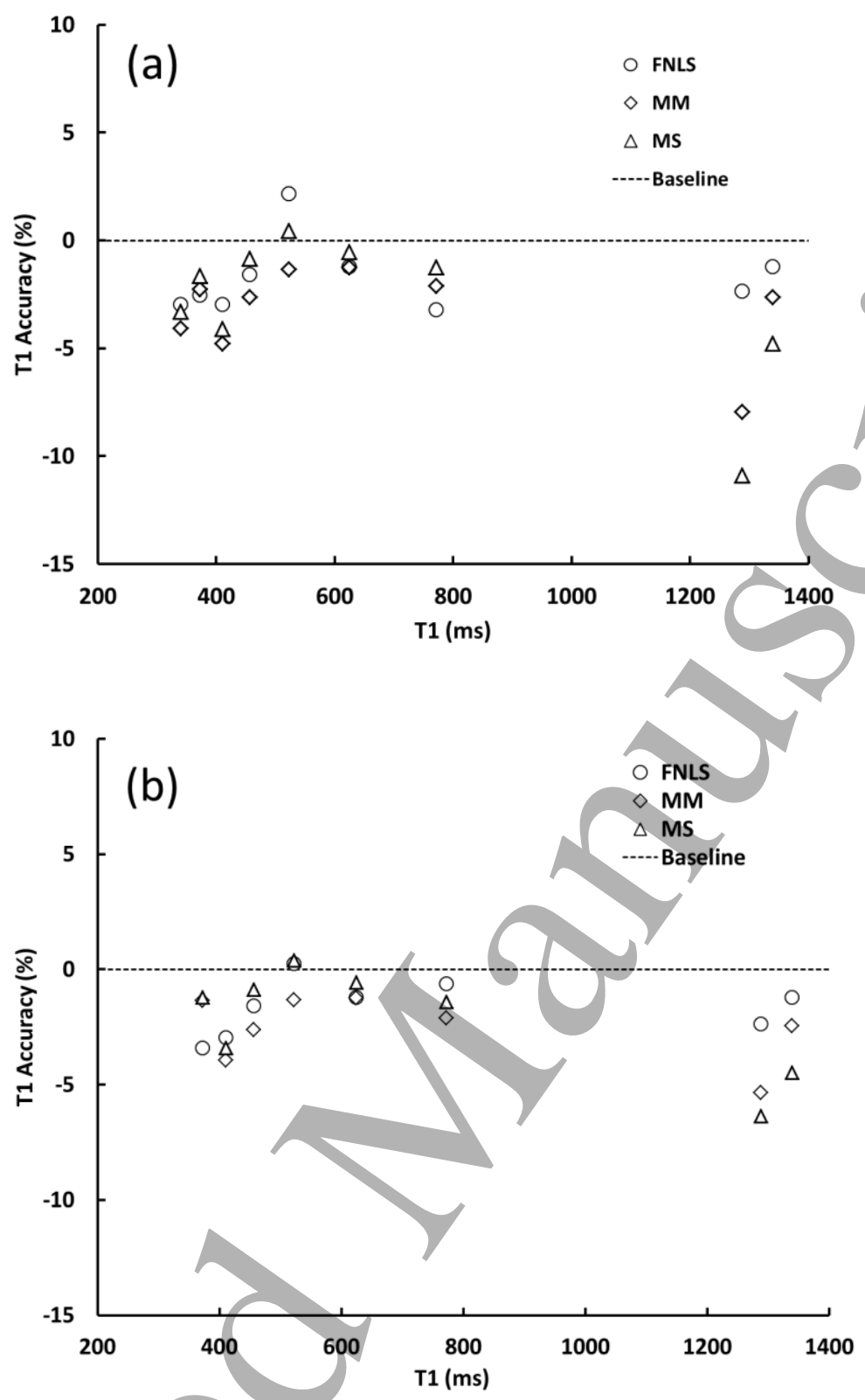

Figure 3. Accuracy of T1 measured by MOLLI sequences for all methods (FNLS, MM and MS) with respect to T1 values obtained by the IR spin-echo sequence: (a) 4(1)3(1)2 sequence, (b) 5(3)3 sequence.

The Gamma analysis of the T1 maps was performed for all the tested methods varying D from $2 \%$ to $5 \%$. An example of this analysis in terms of mean $\gamma$ value $\left(\gamma_{\mathrm{m}}\right)$ and Pass Rate (PR, i.e. the percentage of pixels with $\gamma<1$ ), is reported in Table 1 for the 4(1)3(1)2 sequence (data for the 5(3)3 sequence were almost the same). 
Figure 4(a-c) reports an example of Gamma distributions for the T1 map from the same sequence, obtained with $\mathrm{D}=3 \%$, and referred to FNLS (a) MM (b) and MS (c).

Table 1. Results of the Gamma analysis for different methods, using the numerical phantom as reference $\mathrm{T} 1$ distribution and the T1 maps measured by the 4(1)3(1)2 sequence. The reported $\gamma_{\mathrm{m}}$ (mean $\gamma$ value) and the PR (Pass Rate) were calculated for the three methods varying D from $2 \%$ to $5 \%$. 
(a)

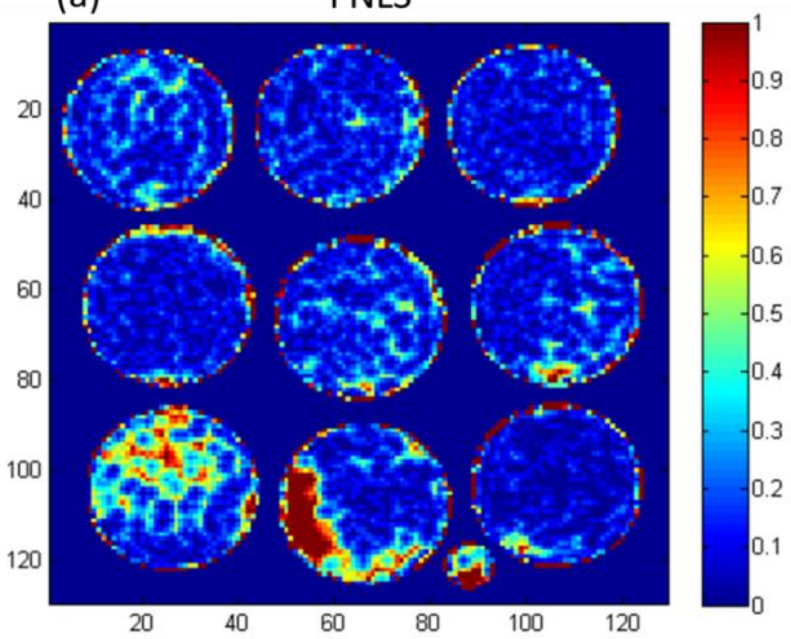

(c)

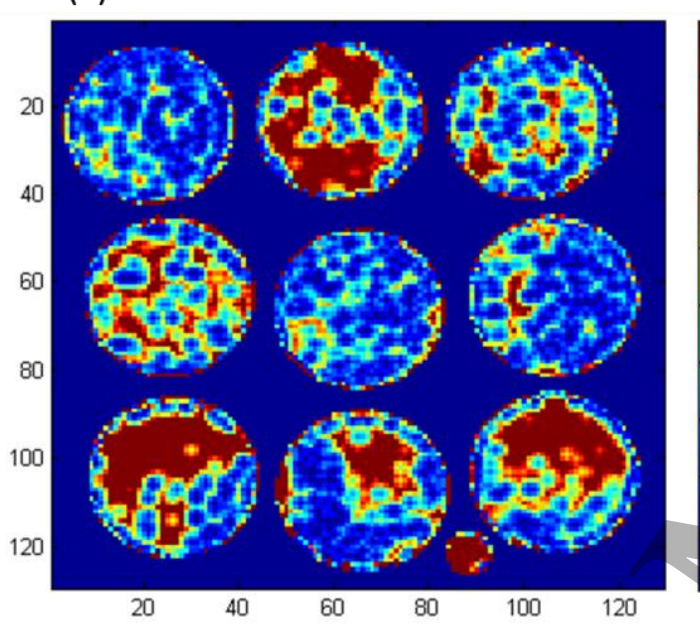

(b)

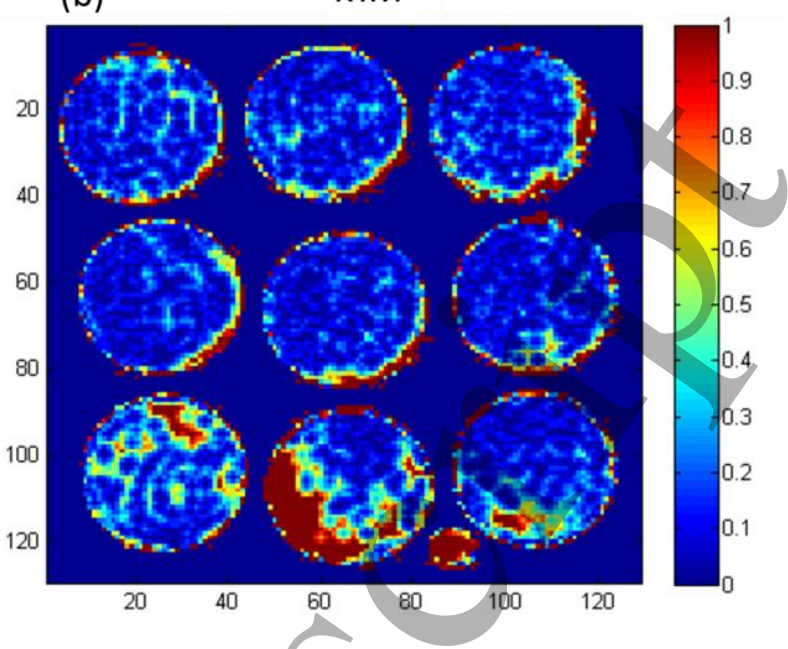

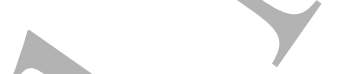

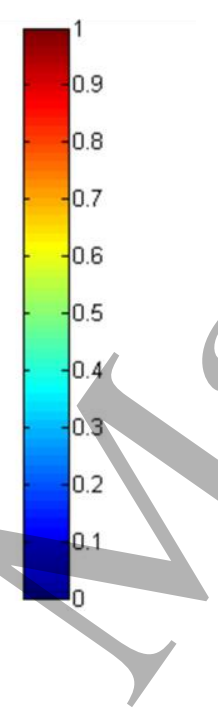

Figure 4. Gamma distributions obtained by the comparison between experimental and numerical (reference) T1 maps: (a) FNLS (b) MM and (c) MS. Tolerance criteria were $\mathrm{D}=3 \%$, and DTA=4.21 $\mathrm{mm}$. The Gamma values are represented with a colorbar.

The uncertainty on the T1 measured by MOLLI was estimated. For the FNLS method, the SD in the ROI on each test object (representing the spatial reproducibility of the T1 value) was at most about $2.4 \%$, similarly to the other methods (up to $2.5 \%$ ). Table 2 summarizes the mean T1 (averaged over repeated measurements) and percent SD for the three methods, obtained by the phantom studies with the 4(1)3(1)2 sequence, repeating the acquisition once a day for 9 days. 
Table 2. Mean T1 (averaged over repeated acquisitions) and corresponding SD, repeating the acquisition with the 4(1)3(1)2 sequence, once a day, for 9 days.

\section{Patient studies}

The mean heart-rate value during the acquisitions was $62 \pm 10 \mathrm{bpm}$. ECVs of healthy and diseased patients are represented in boxplots (Figure 5a-c). The ECV values of the healthy controls ranged for FNLS from $23.4 \%$ to $29.8 \%$ (median value: $25.8 \%$ ), whereas for MM from $24.1 \%$ to $30.1 \%$ (median value: $26.9 \%$ ) and for MS from $23.9 \%$ to $30.6 \%$ (median value: $26.2 \%$ ). For diseased patients, the FNLS yielded ECV values ranging from $31.0 \%$ to $53.9 \%$ (median value: $33.7 \%$ ), $\mathrm{MM}$ values ranged from $28.7 \%$ to $45.9 \%$ (median value: $36.8 \%$ ) and MS values ranged from $31.5 \%$ to $54.9 \%$ (median value: $39.4 \%$ ). The two groups of values significantly differ from each other for all methods. The ECV error was calculated by error propagation for each patient, considering an overall $\mathrm{T} 1$ uncertainty of $10 \%$ (aforementioned). ECV error distributions are reported in Figure 5d-f. The mean relative error on ECV was 2.5\% for FNLS, 2.4\% for MM and 2.3\% for MS. 

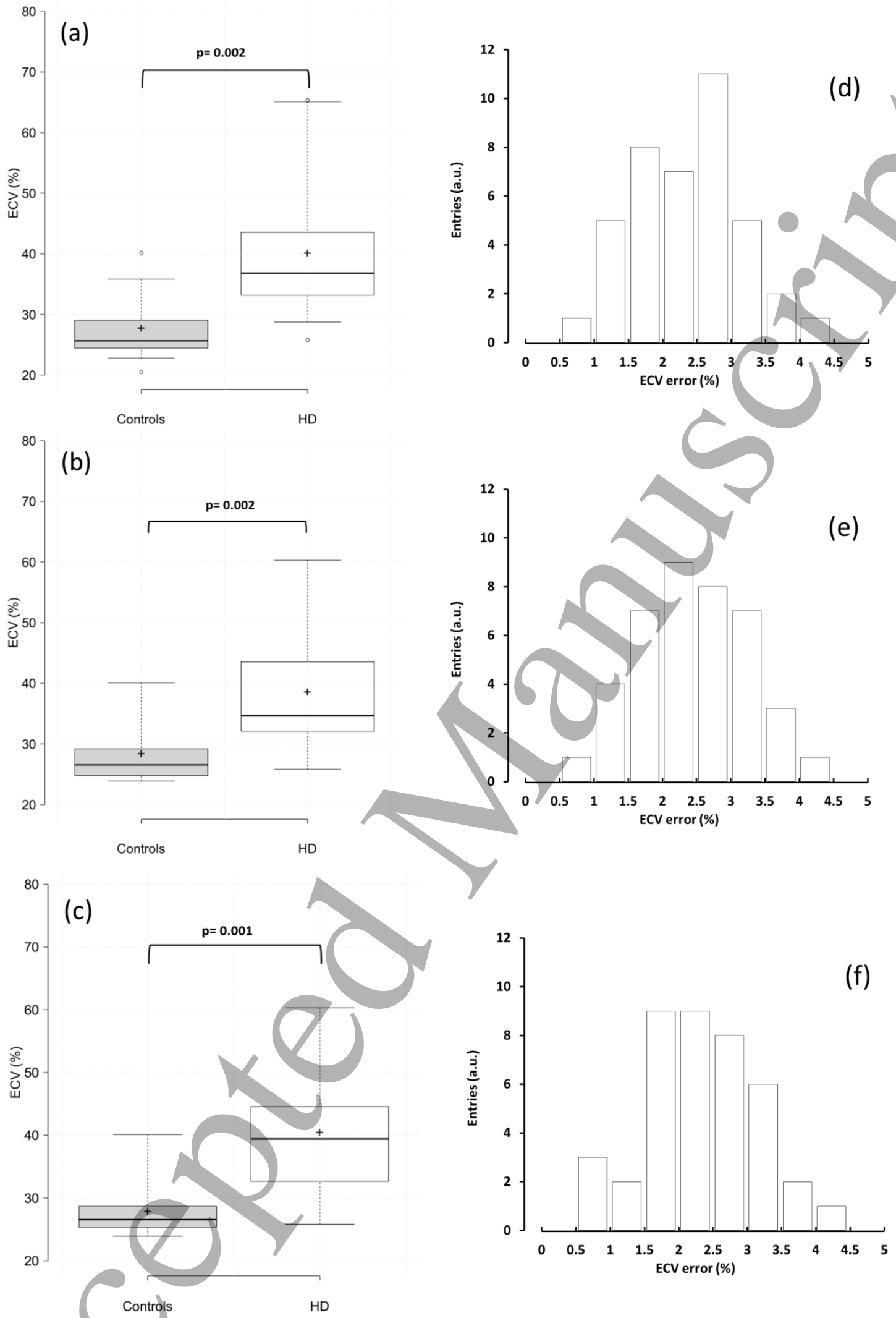

Figure 5. Left panels: boxplot comparing ECV (\%) in controls and diseased (heart disorder, HD) patients for (a) FNLS, (b) MM and (c) MS (p-value of the Kruskal-Wallis test is also reported). Right panel: histograms of the error distribution of the ECV for (d) FNLS, (e) MM and (f) MS. 


\section{Discussion}

Statistical tests and Bland-Altman analysis did not show significant differences among mean T1 datasets from various methods. Also, the estimated uncertainty due to space and time reproducibility for T1 was similar among the methods. The results of the Gamma analysis confirm the similarity of the findings (see Table 1 for a result example). No patterns of disagreement result from a visual analysis of $\gamma$ maps (Figure 4 reports sample data), except for high gradient regions where out-of-tolerance $(>1)$ values at the edges of each flask are systematically present. This is probably due to partial volume effects at the flask edges, reducing the SNR (signal is not present outside the flasks), so noise-related dispersion or bias of the data are more probable. On the other hand, out-of-tolerance values are almost systematic in the vial (where the highest T1 value is present) mainly for MM and MS, and this is also noticeable in plots of T1 accuracy (Figure 3), indicating a better performance for FNLS. Also, the results of the Gamma analysis seems to show slightly better results for FNLS than other methods, as evidenced by lower $\gamma_{\mathrm{m}}$ and higher PR reported in Table 1. A trend T1 underestimation for all methods is also noticeable from Figure 3. This is probably due to linear phase-encode ordering, mostly used for T1 mapping, so the amplitude of the phase-encoding gradient increments in steps. This avoids signal perturbations related to eddy-currents, but on the other hand, it causes a progressive signal saturation related to T2 when approaching the k-space centre [11]. The proposed method reduces the range of search of T1 values, avoid the input of initial parameter, and yield solutions that intrinsically have physical meaning. Consequently, the resulting T1 maps should be less sensitive to the starting seed, and this seems confirmed by the mean accuracy slightly better for the FNLS method (Figure 3). A further benefit of this approach is undoubtedly true: the computational time is at least 25 -fold decreased compared to other (LMbased) methods, making possible also real-time ECV estimation, as well as paves the way to Big Data-related applications [21]. The distribution of the ECV errors (Figure 6) shows a maximum of $4.25 \%$, yielding an experimental uncertainty (with $\mathrm{k}=2$ ) of $8.5 \%$, well below the ECV interpatient variability observed for the healthy subjects $(36.0 \%$ at $2 \mathrm{SD})$, confirming the reliability of diagnosis based on ECV. Myocardial ECV estimations for normal subject were in a good agreement with previous studies that reported values between $25-26 \%[7,22]$. For the diseased subjects examined here the ECV values were in the range $32-54.3 \%$ (median 
2

4

5

6

7

$33.7 \%)$, also in this case in a good agreement with the values reported by previous studies (32.0\%-69.0\%) [22].

\section{Conclusion}

The results obtained with FNLS were indistinguishable from those derived by other well-consolidated methods. However, the new methodology appeared slightly more accurate and more/robust, providing solutions having always a physical meaning. The computational efficiency improves by a factor of at least 25 , making this method more suitable for Big Data related applications.

\section{Acknowledgments}

The authors thank Luisa Altabella for useful discussions and Andreas Greiser (Siemens Healthineers, Erlangen Germany) for the development of Siemens MOLLI sequence prototype and for his constructive support. 


\section{References}

1. Constantine G, et al "Role of MRI in clinical cardiology", Lancet; 363:2162-71 (2004).

2. Kim RJ, et al, "Myocardial gadolinium-DTPA kinetics determine MRI Contrast enhancement and reflect the extent and severity of myocardial injury after acute reperfused infarction", Circulation; 94:3318-26 (1996).

3. Flacke SJ, et al. Measurement of the gadopentetate dimeglumine partition coefficient in human myocardium in vivo: normal distribution and elevation in acute and chronic infarction. Radiology; 218:703-10 (2001).

4. Taylor AJ, et al "T1 mapping: basic techniques and clinical applications", JACC: Cardiovasc Imaging, 9(1): 67-81 (2016).

5. Puntmann VO, et al, "T1 mapping in characterizing myocardial disease: a comprehensive review", Circulation research, 119.2: 277-299 (2016).

6. Iles L, et al, "Evaluation of diffuse myocardial fibrosis in heart failure with cardiac magnetic resonance contrast-enhanced T1 mapping”, J. Am. Coll. Cardiol.; 52:1574-1580 (2008).

7. Dabir D, et al, "Reference values for healthy human myocardium using a T1 mapping methodology: results from the International T1 multicenter cardiovascular magnetic resonance study", J. Cardiovasc. Magn. Reson. 16:69 (2014).

8. Marquardt DW, “An algorithm for least-squares estimation of nonlinear parameters”, J. Soc. Ind. Appl. Math. 11:431-41 (1963).

9. Kammerlander A et al. "T1 mapping by CMR imaging: from histological validation to clinical implication", JACC: Cardiovasc Imaging 9(1):14-23 (2016).

10. Treibel Ta, et al, "Myocardial tissue characterization: histological and pathophysiological correlation", Curr. Cardiovascular Imaging Rep.; 7:1-9, (2014).

11. Cameron D, et al, "Towards accurate and precise $\mathrm{T} 1$ and extracellular volume mapping in the myocardium: a guide to current pitfalls and their solutions", Magn. Reson. Mater. Phy.; doi: $\underline{10.1007 / \mathrm{s} 10334-017-0631-2}$ (2017).

12. Altabella L, et al, "A feasible and automatic free tool for T1 and ECV mapping", Physica Medica; doi: 10.1016/j.ejmp.2016.12.002 (2016).

13. Messroghli DR, et al., "An open-source software tool for the generation of relaxation time maps in magnetic resonance imaging", BMC Med. Imaging 2; 10:16, (2010).

14. Siemens Manual, "Applications Guide: Quantitative Cardiac Parameter Mapping (T1, T2, T2*)", Syngo MR Numaris 4 VB17A (Jan. 2014).

15. Salerno M, et al, "Comparison of Methods for Determining the Partition Coefficient of Gadolinium in the Myocardium Using T1 Mapping”, Magn. Reson. Ima.; 38:217-24 (2014).

16. Barral JK, et al, "A robust methodology for in vivo T1 mapping", Magn. Reson. Med. 64:1057-67 (2010). 
17. Low A and Dempsey JF, "Evaluation of the gamma dose distribution comparison method", Med. Phys. 30, 2455-2464 (2003).

18. Low DA, et al, "A technique for the quantitative evaluation of dose distributions", Med. Phys. 25, 656-661 (1998).

19. Borrazzo C, et al. "Myocardial blood flow estimates from dynamic contrast-enhanced magnetic resonance imaging: three quantitative methods", Phys. Med. Biol.; 63.3: 035008 (2018).

20. Xue H, et al., "Motion correction for myocardial T1 mapping using image registration with synthetic image estimation”, Magn. Reson. Med.; 67:1644-55 (2012).

21. Andreu-Perez, J., et al (2015). Big data for health. IEEE (2015) J Biomed Health Inform, 19(4), 11931208.

22. Kellman P, et al., "Extracellular volume fraction mapping in the myocardium, part 2: initial clinical experience”, J. Cardiovascular Magn. Reson. 14, 64 (2012). 